\title{
Posibilidad, realidad y necesidad del discurso práctico
}

\author{
Possibility, reality and need for practical discourse
}

Alberto Damiani*

Resumen: El propósito de este trabajo es explicar la relación entre acción, reglas y discurso. El trabajo comienza con una presentación de algunas objeciones a la ética del discurso y su base común: la independencia entre moralidad y razón. Luego son propuestas contra esas objeciones dos tesis sobre la relación entre acción y discurso en un marco pragmático trascendental. La conclusión es que la posibilidad del discurso es una condición del sentido de la acción humana.

Palabras clave: De Zan, regla, norma, discurso, acción

\begin{abstract}
The aim of this paper is to explain the relation between action, rules and discourse. The paper begins with a presentation of some objections to the Discourse Ethics and their common basis: the independence between morality and reason. After that, against these objections are proposed two theses about the relation between action and discourse in a transcendental-pragmatic frame. The conclusion is that the possibility of discourse is a condition of the sense of human action.
\end{abstract}

Key words: De Zan, rule, norm, discourse, action

Desde el año 2006 se realizaron en la Fundación ICALA de la ciudad de Río Cuarto una serie de coloquios anuales sobre Ética del Discurso, en los que participamos un conjunto de colegas, argentinos y extranjeros, interesados en el tema. Tuvimos la suerte de que el Profesor De Zan

\footnotetext{
* Doctor en Filosofía por la UBA, ha obtenido la Habilitation für Philosophie de la Freie Universität Berlin. Profesor Titular regular de Historia de la Filosofía Moderna en la UBA y en la UNR, Profesor Adjunto regular a cargo de Filosofía Política en la UBA e Investigador Principal del CONICET. Director del Centro de Estudios en Filosofía Moderna de la UNR. Director del Departamento de Filosofía de la UBA. Director de la Sección de Filosofía Política y Social del Instituto de Filosofía de la UBA. Dirección electrónica: damial@,filo.uba.ar
} 
presentara allí varias contribuciones, y de que discutiera las tesis y los argumentos de los demás participantes. En el presente artículo quisiera ofrecer una perspectiva que ayude a responder dos preguntas inquietantes, formuladas por el profesor De Zan como títulos de dos de sus contribuciones: ¿Está agotado el programa de investigación de la ética del discurso? y ¿Queda algo de la herencia kantiana en la ética discursiva de Habermas?

En el primer trabajo indicado, De Zan presenta a la Ética del Discurso como un prometedor programa de investigación filosófica, que necesita redefiniciones, complementaciones y nuevos desarrollos. El punto de partida de la realización de estas tareas es el reconocimiento de las condiciones históricas y teóricas de esta concepción ético-filosófica. Entre las primeras se mencionan "el contexto de la cultura ilustrada y de la sociedad moderna, en la que está reconocido el derecho de la conciencia moral autónoma al cuestionamiento reflexivo de valores o de normas tradicionales y solamente la justificabilidad racional de los mismos vale como fuente de legitimación racional y política" (p. 14). Entre las segundas, las teorías del lenguaje, de la racionalidad y de la acción, que ofrecen el vocabulario para formular el programa de investigación de la Ética del Discurso.

Como es sabido, Karl-Otto Apel y Jürgen Habermas ofrecen versiones distintas de ese programa. De Zan advierte que la diferencia fundamental entre estos autores no se reduce al conocido tópico de la fundamentación última de la ética, sino que se extiende al "concepto de la filosofía y de su función en la cultura contemporánea" (p. 18). Si bien De Zan lamenta que Apel se abroquele en una concepción de la filosofía primera como semiótica trascendental, reconoce que la acertada tesis de Habermas sobre el parasitismo de la racionalidad estratégica respecto de la racionalidad comunicativa no resulta suficiente en un contexto de interacción social abiertamente estratégica. La rectificación de la Ética del Discurso, propuesta

${ }^{1}$ De Zan, Julio, “¿Está agotado el programa de investigación de la ética del discurso?”, en: D. J. Michelini, R. Maliandi, J. De Zan (eds.), Ética del discurso, recepción y críticas desde América Latina, Río Cuarto, Ediciones del Icala, 2007, pp. 13-45; De Zan, Julio, “QQueda algo de la herencia kantiana en la ética discursiva de Habermas?”, en: D. J. Michelini, J. De Zan, A. M. Damiani (eds.), Ética, politicay discurso, Río Cuarto, ICALA, 2015, tomo 1, pp. 162-172. 
por De Zan, remite a un ejercicio de articulación de la misma con las teorías del reconocimiento intersubjetivo y con los puntos de vista del realismo filosófico político, que él desarrolla exhaustivamente en otros textos. ${ }^{2}$

En el tratamiento de la herencia kantiana de Habermas, De Zan advierte que, en el Prólogo de la Grundlage, Kant diferencia dos niveles de la reflexión moral: un nivel puro de la fundamentación a priori del principio universal de la moralidad y un nivel de aplicación de ese principio al examen de las máximas particulares y del juicio sobre la acción en las diferentes situaciones concretas. Esta diferencia kantiana es oscurecida por Habermas, quien pretende realizar ambas operaciones mediante el mismo procedimiento de los discursos morales empíricos, semejantes al procedimiento del juicio reflexionante, que Kant desarrolla en su teoría del juicio estético y aplica en la segunda parte de su Crítica de la razón práctica. Cuando en Facticidad y validez, el principio del discurso es redefinido como anterior a la diferenciación de moral, derecho y política, Habermas expande a todo el ámbito de la filosofía práctica las consecuencias de su clasificación de las formas de la racionalidad de la interacción social y de su tesis sobre el parasitismo de la forma estratégica respecto de la comunicativa. De Zan aclara esta tesis del siguiente modo:

si el predominio de la lógica de la razón instrumental y estratégica desactivara o anulara toda racionalidad comunicativa, entraría finalmente en crisis también la propia racionalidad estratégica, es decir, quedaría fuera de juego toda racionalidad, y se habría retrocedido por detrás de lo político y de la sociedad civil, a la situación de estado de naturaleza. ${ }^{3}$

En lo que sigue, quisiera formular algunas aclaraciones referentes a la relación entre los distintos tipos de acción, las reglas que las rigen y los tipos de discurso. Estas aclaraciones me permitirán mostrar que el discurso no se reduce ni a un medio de resolución de pretensiones de validez, ni a un mecanismo de coordinación de interacciones sociales, sino que su posibilidad

${ }^{2}$ De Zan, Julio, La vieja y la nueva política. Libertad, poder y discurso, Buenos Aires/San Martín, Jorge Baudino Editores/UNSAM Edita, 2013.

${ }^{3}$ De Zan, Julio, "¿Queda algo de la herencia kantiana en la ética discursiva de Habermas", p. 171. 
constituye una condición de sentido de las acciones humanas en general. Para cumplir con este objetivo comenzaré presentando, por un lado, algunos presupuestos normativos irrebasables, explicitados y probados mediante la reflexión pragmático-trascendental y, por el otro, el núcleo común de algunas objeciones a la ética del discurso: la independencia recíproca entre moralidad y racionalidad (1). Luego sostendré, por un lado, que todas las acciones humanas son acompañadas tácitamente por la posibilidad de un discurso en el que se puedan resolver las pretensiones de validez implícitas en las reglas que las rigen, y, por el otro, que todo actor es un potencial interlocutor que puede participar en ese discurso (2).

En la formulación de estas aclaraciones me propongo seguir el método dialógico reflexivo que aprendimos del profesor De Zan en los mencionados coloquios de Río Cuarto, con la esperanza de contribuir al programa de investigación filosófica abierto por la Ética del Discurso.

1. La ética normativa es una disciplina filosófica que se ocupa de considerar críticamente las normas morales admitidas como vinculantes por los agentes de distintas comunidades humanas. Uno de los problemas examinados por la ética normativa es el de la fundamentación racional de dichas normas. Este problema consiste en determinar, en cada caso, si hay razones que justifiquen la validez de las obligaciones morales fácticamente vigentes en el ethos. La ética del discurso es una propuesta de ética normativa que intenta resolver este problema, distinguiendo dos niveles de análisis. En el primer nivel, el discurso práctico es presentado como el único medio disponible para determinar las razones que justifican la validez de las normas morales. En el segundo nivel, el discurso práctico es examinado reflexivamente, para hallar las razones que lo fundamentan. Este examen reflexivo permite, en primer lugar, descubrir algunas reglas presupuestas en la parte performativa de los actos que componen el discurso práctico y, en segundo lugar, mostrar que las mismas no pueden negarse sin inconsistencia pragmática y no pueden deducirse sin circularidad lógico-pragmática. Algunas de estas reglas tienen un significado moral, por ejemplo, el denominando principio procedimental o metanorma del discurso práctico.

La propuesta de solución al problema de la fundamentación filosófica de la moralidad, ofrecida por la ética del discurso, ha recibido varias objeciones derivadas de la presunta distancia entre el discurso racional y la 
moralidad de las acciones. Los críticos de la ética del discurso creen encontrar esa distancia en la diferencia básica entre las reglas necesariamente presupuestas en el discurso práctico y las normas morales que deben regir las acciones humanas. Esta diferencia consiste en lo siguiente. El discurso se compone de actos de habla que presuponen condiciones necesarias y constitutivas de su sentido, algunas de las cuales parecen tener un carácter éticonormativo. Por ejemplo, las siguientes: no excluir arbitrariamente o discriminar a las personas, resolver sus controversias mediante argumentos y reconocerlas iguales en derechos y deberes. Estas condiciones son necesarias y constitutivas porque, si no se cumplen, los actos que componen un discurso práctico no pueden realizarse; en términos de John Austin, son desafortunados. Sin embargo, una característica distintiva de una norma moral consiste en que su cumplimiento depende exclusivamente del arbitrio del agente. El concepto de una obligación moral remite a una situación en la que el actor puede decidir libremente si realiza una acción prescripta por una norma. Alguien es, entonces, responsable por una acción sólo si la ha realizado libremente. Por lo tanto, a diferencia de lo que ocurre con las reglas constitutivas, las obligaciones derivadas de las normas morales no siempre son cumplidas, sino que siempre deben ser cumplidas.

Esta diferencia conceptual entre las reglas constitutivas del discurso y las normas morales ha servido como ocasión para formular, al menos, tres objeciones a la ética del discurso. La primera objeción se ha presentado como el reproche de cometer una "falacia intelectualista", consistente en pretender fundamentar las normas morales, obligatorias en la praxis, sobre la única base de principios racionales, tales como las condiciones necesarias de la argumentación. ${ }^{4} \mathrm{La}$ segunda objeción concede que los presupuestos constitutivos del discurso no pueden ser cuestionados sin autocontradicción performativa, pero rechaza que se les atribuya un contenido moral o que de ellos pueda extraerse un principio ético-normativo o meta-norma procedimental del discurso. ${ }^{5}$ La tercera objeción advierte que aunque los interlocutores discursivos estuvieran obligados por presupuestos ético-

\footnotetext{
4 Ilting, Karl-Heinz, Grundfragen der praktischen Philosophie, Frankfurt a. M., Suhrkamp, 1994.

5 Wellmer, Albrecht, Ethike und Dialog. Elemente des moralischen Urteils bei Kant und in der Diskursethik, Frankfurt a. M., Suhrkamp, 1986.
} 
normativos del discurso, podrían librarse de esa exigencia cuando salieran del círculo de la argumentación. ${ }^{6}$

El mérito de estas objeciones consiste en señalar la necesidad de aclarar el hecho de que algunas reglas constitutivas del discurso puedan ser también moralmente obligatorias. ${ }^{7}$ En este trabajo quisiera señalar un error común a las tres objeciones. El mismo radica en presuponer dogmáticamente que las acciones humanas son totalmente independientes del discurso práctico y que, por tanto, la moralidad de dichas acciones es independiente de su racionalidad. Contra este presupuesto intentaré mostrar, en el presente trabajo, una estrecha conexión entre acción y discurso, que puede expresarse mediante las dos formulaciones siguientes. Toda acción es acompañada necesariamente por la posibilidad de un discurso sobre ella. Todo actor es, necesariamente, un potencial interlocutor discursivo, capaz de participar en la resolución argumentativa de las pretensiones de validez implícitas en sus acciones. Creo que estas dos formulaciones agotan el significado del título que elegí para este trabajo: la necesaria posibilidad del discurso. El mismo indica que el discurso no es sólo algo contingente, que puede o no ocurrir fácticamente, sino que su posibilidad es una condición constitutiva del sentido de la acción humana. A mi juicio, una comprensión filosófica del concepto de acción debe explicitar esta necesaria posibilidad del discurso contenida en toda acción.

Para obtener esa comprensión filosófica es necesario evitar dos tipos de reduccionismo, usuales en las actuales teorías filosóficas de la acción humana. El primero se deriva de la propuesta de organizar la diversidad de tipos de comportamiento humano tomando como unidad de análisis las denominadas "acciones básicas" (basic actions), es decir, los movimientos corporales mediante los que serían ejecutadas otras acciones más complejas. Estas acciones básicas son el punto de partida para la formulación de una serie de descripciones e interpretaciones de la acción, ordenadas en una secuencia

${ }^{6}$ Habermas, Jürgen, Moralbewnsstsein und kommunikatives Handeln, Frankfurt a. M., Suhrkamp, 1983, p. 96.

7 Damiani, Alberto, "Reglas discursivas y normas morales", Revista de Filosofia, 41, 1 (2016), pp. 7-31. 
de complejidad creciente. ${ }^{8}$ Contra esta propuesta se ha advertido que los movimientos corporales no pueden identificarse propiamente con acciones, sino que resulta preferible concebirlos, o bien como comportamientos coejecutados con las acciones, o bien como un aspecto de la infraestructura de las mismas. ${ }^{9}$

Un segundo tipo de reduccionismo usual en las actuales teorías filosóficas de la acción consiste en recurrir al modelo de la observancia consciente de una regla. ${ }^{10}$ Mediante este recurso, la observancia de una regla de cálculo aritmético o de una regla gramatical para el uso de un predicado en un lenguaje natural es propuesta como una matriz conceptual para comprender el sentido de las acciones humanas regidas por normas socialmente aceptadas. Contra este recurso, se ha observado, hace ya tiempo, que existen significativas diferencias entre las reglas operacionales (por ejemplo, aritméticas, lógicas o gramaticales) y las reglas prácticas, que rigen las acciones humanas en sentido estricto. En el marco de este trabajo quisiera recordar sólo la siguiente diferencia. Las reglas operacionales permiten al agente realizar una operación y al observador comprenderla, o sea, identificarla como una operación particular, distinta de otras. Pero estas reglas no le permiten al observador explicar por qué el agente realiza esa operación en una situación determinada, ni le permiten al agente alegarlas como razones que justifiquen la realización de la misma. ${ }^{11}$

Una vez diferenciado el concepto de acción humana de los movimientos corporales y de las operaciones, es posible advertir que las

${ }^{8}$ Danto, Arthur, Analytical Philosophy of Action, Cambridge, Cambridge University Press, 1973; Davidson, Donald, Essays on Actions and Events, Oxford, Oxford Clarendon Press, 1980; Feinberg, Joel, "Acción y responsabilidad", en: A. R. White (ed.), La filosofía de la acción, México, FCE, 1976, pp. 139-174.

${ }^{9}$ Goldmann, Alvin, A Theory of Human Action, Engelwoood Cliffs, NJ, Prentice-Hall, 1970; Habermas, Jürgen, "Handlungen, Operationen, körperliche Bewegungen”, en: id., Vorstudien und Ergänzungen zur Theorie des kommunikativen Handelns, Frankfurt a. M., Suhrkamp, 1984, pp. 273-306.

${ }^{10}$ Winch, Peter, The Idea of Social Science and Its Relation to Philosophy, London, Routledge and Kegan Paul, 1958; von Wright, Georg, Norm and Action, London, Routledge and Kegan Paul, 1963.

${ }^{11}$ Habermas, Jürgen, "Handlungen...", op. cit. 
acciones humanas están regidas por cierto tipo muy específico de reglas, gracias a las cuales los agentes pueden no sólo identificar y hacer comprensibles sus acciones, sino también intentar dar razón de ellas. La actividad de dar razones para justificar una acción no es homogénea, sino que sus características dependen del tipo de acción específica que se realice. Para reconstruir esta diversidad de tipos de acción, en relación con las reglas que siguen y que pueden justificarlas, puede adoptarse la clasificación de acciones propuesta por Jürgen Habermas, es decir: acciones instrumentales, estratégicas y comunicativas. ${ }^{12}$ Una acción instrumental está orientada por una regla técnica, que prescribe los medios adecuados para manipular objetos según un propósito subjetivo del agente. Una acción estratégica está orientada por una regla que prescribe los medios adecuados para influir en las decisiones de adversarios con los que el agente compite. En estos dos casos, la regla de acción será una buena razón para actuar sólo si expresa un conocimiento verdadero de regularidades empíricas naturales, en el primer caso, y sociales, en el segundo. Por último, si se trata de una acción comunicativa, estará orientada por una norma que regula las expectativas comunes y recíprocas de los participantes de una interacción social cooperativa. En este caso, la norma es una buena razón para actuar sólo si es racionalmente admisible para los afectados por esa acción. En los tres tipos de acción, por tanto, las reglas prácticas permiten no sólo, como las reglas operacionales, identificar y hacer comprensibles los comportamientos que regulan, sino también dar razones de la realización de las mismas.

Llegados a este punto, podemos advertir que las acciones humanas pueden ser concebidas como un tipo de comportamiento regido por cierta clase específica de reglas, que podemos denominar "reglas prácticas". La especificidad de estas reglas radica en que pueden ser utilizadas como razones, en el procedimiento de justificación racional de las acciones que regulan. Dicho en otros términos: la posibilidad de un discurso sobre una acción se encuentra siempre ya disponible en la regla que la rige, porque esa regla puede alegarse en el discurso como razón para justificar esa acción. Para aclarar esta posibilidad necesaria del discurso podemos considerar una acción instrumental mediante la cual un agente intenta modificar un estado de cosas para realizar un

12 Habermas, Jürgen, Theorie des kommunikativen Handelns, Frankfurt a. M., Suhrkamp, 1981, Bd. 1, pp. 369-452. 
propósito subjetivo; por ejemplo, la producción de un artefacto siguiendo las instrucciones de un manual. Si un interlocutor le preguntara a ese agente por qué realiza esa acción, la respuesta podría ser la explicitación del propósito ("quiero producir ese artefacto") y de la regla técnica, contenida en el manual, que conecta el fin propuesto con la acción instrumental realizada como medio para realizar ese fin. El interlocutor podría rechazar esta respuesta como inválida, objetando la pretensión de verdad del enunciado causal, implícito en la regla técnica, que conecta la acción, como causa, con el estado de cosas buscado, como efecto. Quien realiza una acción instrumental eleva, por tanto, una pretensión de verdad, que puede ser resuelta mediante un discurso teórico en el que podría participar como interlocutor.

También podemos considerar el ejemplo de una acción estratégica mediante la cual un agente intenta influir en otros agentes en una interacción social competitiva para maximizar su beneficio; por ejemplo, la denuncia pública de una incapacidad de un adversario político para quitarle la adhesión del electorado. Si un interlocutor le preguntara por qué realiza esa acción, la respuesta podría ser la explicitación del propósito de ganar la competencia electoral y de cierta regla que lo conecte con la denuncia realizada. El interlocutor podría rechazar esta respuesta como inválida, objetando la pretensión de verdad del enunciado causal, implícito en la regla técnica, que conecta la acción, como causa, con el estado de cosas buscado, como efecto, por ejemplo, advirtiendo que al electorado no le interesa semejante incapacidad del adversario. Por lo tanto, quien realiza una acción estratégica también eleva, implícitamente, una pretensión de verdad, que puede ser resuelta mediante un discurso teórico en el que podría participar como interlocutor.

Algo semejante ocurre con las denominadas acciones comunicativas, donde no encontramos actores solitarios que pretenden realizar sus propósitos subjetivos para transformar un estado de cosas natural o para influir sobre las acciones de sus competidores, sino con los miembros de un grupo social que regulan sus comportamientos según normas morales. Estas normas expresan las expectativas generalizadas de comportamiento de los miembros de ese grupo, quienes pueden observarla o violarla cuando se dan las condiciones a que la norma se aplica. Si, en este caso, un interlocutor le preguntara al actor por qué realiza esa acción, la respuesta podría ser la 
explicitación del propósito de cumplir con una norma aceptada por su comunidad. El interlocutor podría rechazar esta respuesta como inválida, en dos sentidos diversos. Por un lado, objetando la pretensión de verdad del enunciado que afirma la existencia, en la situación en cuestión, de las condiciones necesarias y suficientes de la aplicación de esa norma. Por otro lado, objetando la pretensión de justicia de dicha norma, esto es, impugnarla como moralmente incorrecta. Por lo tanto, quien realiza una acción comunicativa en un marco de interacción cooperativa eleva, implícitamente, una pretensión de verdad, que puede ser resuelta mediante un discurso teórico, y también una pretensión de justicia, que puede ser resuelta mediante un discurso práctico. En ambos discursos podría participar como interlocutor el agente al que se le imputa la autoría de la acción.

En este punto quisiera formular dos aclaraciones. En primer lugar, no siempre hay necesidad de llevar adelante estos discursos teóricos sobre las pretensiones de verdad contenidas en los enunciados causales, implícitos en las reglas técnicas y estratégicas y en la afirmación sobre las condiciones de aplicación de las normas morales. Tampoco hay necesidad de llevar adelante discursos prácticos sobre las pretensiones de justicia contenidas en las normas que regulan las expectativas generalizadas de comportamiento en un grupo social. Recién cuando son cuestionadas dichas pretensiones de validez implícitas en las reglas prácticas, aparece la exigencia de implementar realmente un discurso para resolverlas a favor o en contra. En segundo lugar, tanto el discurso teórico como el discurso práctico, que permiten resolver las pretensiones de validez implícitas en la regla que sigue el actor, pueden darse sin la participación efectiva del actor. Sin embargo, lo que aquí nos interesa no es ni la realización fáctica de dicho discurso, ni la participación fáctica del actor en el mismo, sino lo que puede expresarse mediante las dos afirmaciones siguientes: (1) la posibilidad del mencionado discurso es una condición necesaria del sentido de la acción, sin la cual no puede ser comprendida, ni realizada, y (2) la posibilidad de la participación del actor en dicho discurso es una condición necesaria de su rol de actor, es decir, sin ella no podríamos imputarle a él dicha acción como su acción. O, dicho de otra manera: no hay acción con sentido sin la posibilidad de un discurso sobre ella y no hay actor que no pueda ser considerado como un interlocutor que participe de ese discurso. 
2. El discurso no puede reducirse a un conjunto de operaciones lingüísticas que tiene lugar cuando se interrumpe una acción, para examinar críticamente las reglas prácticas que la regulan y la información sobre el mundo natural y social presupuesta por dichas reglas. Además de esta alternancia fáctica entre acción y discurso, puede reconocerse una conexión estrecha, una unidad indisoluble entre ellos si se atiende al discurso que debe poder acompañar a toda acción, de manera análoga a cómo, según Kant, el yo pienso debe poder acompañar a todas mis representaciones. ${ }^{13}$ Quisiera, entonces, destacar del siguiente modo esta diferencia entre el discurso real y el discurso posible. Si bien el discurso real es una actividad alternativa a la acción, mediante la cual puede planearse y coordinarse la acción, el discurso posible es una condición necesaria del sentido de la acción, o para decirlo en términos kantianos, una condición trascendental de la acción. Las objeciones a la ética del discurso mencionadas al comienzo parecen haberse concentrado exclusivamente, por tanto, en la implementación de los discursos reales y haber ignorado, o al menos descuidado, el discurso posible implícito en toda acción.

De la misma manera, puede reconocerse que la participación en el discurso no puede reducirse a las operaciones lingüísticas realizadas efectivamente por los interlocutores que se proponen examinar críticamente las pretensiones de validez implícitas en las reglas prácticas que regulan las acciones realizadas por los actores. Estas operaciones lingüísticas, realizadas por quienes efectivamente participan de un discurso, son distintas de las acciones que el mismo tiene por objeto. Los actores pueden participar o no participar en los discursos mediante los que se intenta resolver las pretensiones de validez implícitas en sus acciones. Pero lo que interesa destacar aquí es que además de esta participación contingente de los actores en los discursos reales, puede reconocerse, en general, una unidad indisoluble entre el rol del interlocutor discursivo y el rol del actor en el mundo de la vida. Para reconocer esta unidad necesaria de roles basta con advertir que todo actor es necesariamente un potencial interlocutor, alguien que debe poder responder por sus acciones, es decir, contribuir a la resolución discursiva de las pretensiones de validez implícitas en las reglas que las regulan.

13 Kant, Immanuel, Kritik der reinen Vernunft (1787), en: Kants gesammelte Schriften. Akademie-Textausgabe, Berlín, 1968, B131-136, § 16. 
Para comprender esta unidad entre acción y discurso les propongo detenernos un momento en la caracterización de los dos roles mencionados. Las acciones humanas, a diferencia de cualquier fenómeno natural, pueden ser consideradas no sólo desde la perspectiva de un observador que intenta describirlas y explicarlas objetivamente, sino también desde la perspectiva del participante de la interacción social, comprometido con la situación, los intereses y las relaciones sociales en las que le toca actuar. Puede denominarse a este rol de participante como el del actor en el mundo de la vida. Por otro lado, este participante de la interacción social puede asumir también otro rol, en cuanto se plantea alguna cuestión de validez respecto del curso de acción elegido por alguno de los sujetos que interactúa. Este otro rol puede denominarse como el del interlocutor discursivo.

A fin de aclarar esta diferencia de roles, es necesario advertir que la misma no debe pensarse atendiendo a una situación en la que un individuo actúa y otro se plantea cuestiones de validez respecto del curso de acción elegido por el primero. Se trata más bien de dos actitudes que puede asumir, sucesiva o simultáneamente, un mismo individuo en una misma interacción social. En ese sentido, estos dos roles no son sólo distintos sino también inseparables y complementarios. Ello es así, por un lado, porque quien participa del discurso para contribuir a resolver colectivamente un problema teórico o práctico realiza actos de habla; por ejemplo: preguntar, responder, afirmar, objetar, fundamentar, etc. Es decir que el discurso es un tipo especial de interacción social y el interlocutor que participa en el discurso es ya un actor, porque contribuye, mediante los actos mencionados, a la resolución discursiva de un problema teórico o práctico. En rigor la figura del observador que pretende sólo describir o explicar objetivamente una acción es una representación deficitaria del sujeto cognoscente, deudora de la idea clásica de theoría, como contemplación pasiva. El déficit de esta representación reside en que la adquisición de conocimiento supone necesariamente la realización de ciertos actos, a saber, los actos que realizamos cuando participamos en un discurso. Si se atiende a estos actos, se advierte que quien conoce actúa, o mejor dicho interactúa, junto con otros en un proceso discursivo. La idea clásica de theoría hace abstracción de estas operaciones discursivas, imprescindibles para el conocimiento humano. 
Por otro lado, los dos roles mencionados son inseparables y complementarios también por otra razón que es especialmente relevante para el objetivo de este trabajo: el actor del mundo de la vida siempre puede devenir interlocutor discursivo que contribuya a resolver las pretensiones de validez implícitas en su acción. Consideremos esta posibilidad más de cerca. Quien simplemente actúa, es decir, quien participa de la interacción no discursiva, mediante acciones lingüísticas y no lingüísticas, tanto estratégicas como comunicativas, no desempeña fácticamente el rol de interlocutor discursivo. Se trata del caso de quien no se detiene a proponer ante otros su curso de acción, ni formula explícitamente una pretensión de validez que los otros pueden evaluar y, por lo tanto, tampoco ofrece argumentos para justificar racionalmente su pretensión y responder a las objeciones de sus interlocutores. Un aspecto decisivo de este rol de simple actor en el mundo de la vida consiste, sin embargo, en que quien lo desempeña, aunque no se detiene a justificar racionalmente sus acciones, podría hacerlo. Es decir, todo actor es potencialmente un interlocutor discursivo.

Este segundo aspecto de la conexión entre los dos roles mencionados reside, entonces, en que la posibilidad de participar en el discursivo se encuentra necesariamente implícita en el concepto de actor humano en general. Quien simplemente actúa sin argumentar para justificar racionalmente su comprensión de la situación, la conveniencia de los medios empleados para realizar un objetivo o la corrección moral de su acción, podría sin embargo argumentar para ello. Esta posibilidad no actualizada fácticamente puede presentarse, por ejemplo, en el caso de quien simplemente actúa y, pudiendo argumentar, no lo hace porque las circunstancias no parecen exigirlo o porque quienes interactúan con él no encuentran motivos para exigirle razones. Esta posibilidad puede pensarse también en el caso de quien, encontrándose con la exigencia de justificar la corrección moral de su acción, prefiere no hacerlo, sea porque no cree que haya razones suficientes para esa empresa, sea porque no cree contar en un momento dado con esas razones, sea porque las circunstancias parecen impedirle la implementación de un discurso práctico mediante el que se resuelvan sus pretensiones de corrección normativa. También puede pensarse en el caso de quien fácticamente no es capaz de actualizar la posibilidad mencionada y otros casos en los que un actor del mundo de la vida no actualiza la posibilidad de justificar racionalmente un curso de acción que él mismo ha elegido. Independientemente de esa variedad 
de casos, es necesario destacar aquí que toda acción en el mundo de la vida puede ser juzgada, como un medio adecuado para alcanzar un fin o como moralmente correcta e incorrecta y que el discurso, teórico o práctico respectivamente, es el único medio para justificar racionalmente dicho juicio.

Para evitar el error cometido por las mencionadas objeciones a la ética del discurso, basta con reconocer que el sentido de las acciones humanas en general depende del discurso y que, por tanto, la moralidad de dichas acciones depende de su racionalidad. Una condición de la posible implementación de un discurso práctico efectivo, en el que se resuelvan las pretensiones de corrección normativa, consiste justamente en el discurso que implícita y virtualmente acompaña la realización de toda acción humana en el mundo de la vida. ${ }^{14}$ Toda acción humana se encuentra orientada por el propósito del agente y este propósito presupone necesariamente cierta (pre-)comprensión del agente sobre la situación en la que le toca actuar. Es cierto que un agente puede simplemente actuar, sin presentar ante posibles interlocutores su propósito y su comprensión e, incluso, sin reflexionar en soledad sobre los mismos. Sin embargo, lo que interesa destacar aquí es que tanto el propósito del agente como su comprensión de la situación pueden ser objeto de un discurso, en el que se expliciten y evalúen las pretensiones de validez implícitas en el propósito y la comprensión que orientan y motivan la acción del agente. En otros términos, toda acción humana se realiza acompañada de la posibilidad de dos discursos. Por un lado, un discurso práctico en el que podría examinarse la pretensión de rectitud normativa implícita en el propósito que motiva la acción y en la norma o máxima que la orienta. Por otro lado, un discurso teórico en el que podría examinarse la pretensión de verdad implícita en la comprensión que el agente tiene de la situación en la que actúa y de los conocimientos presupuestos en las reglas técnicas o estratégicas que orientan su acción.

Esta idea de un discurso implícito acompañante de toda acción humana resulta fundamental para comprender la unión indisoluble de los roles del interlocutor discursivo y del actor, que puede formularse del siguiente modo:

14 Böhler, Dietrich, "Handlung und Begleitdiskurs oder Selbsteinholung und Mitverantwortung des leibhaften Diskurspartners", en: J. O. Beckers, F. Preußger, Th. Rusche (Hrsg.), Dialog, Reflexion, Verantwortung. Zur Diskussion der Diskurspragmatik, Würzburg, Könighausen \& Neumann, 2013, pp. 3-46. 
el actor del mundo de la vida es ya un posible interlocutor. El propósito que motiva su acción, su comprensión de la situación en la que actúa y la regla que la orienta contienen implícitamente pretensiones de validez que sólo pueden ser resueltas mediante un discurso. Si dicho propósito y dicha comprensión fuesen enunciados explícitamente mediante actos de habla regulativos y constatativos respectivamente, estos actos contendrían en su parte performativa pretensiones de validez que sólo podrían resolverse mediante el discurso.

Este concepto de un discurso virtual acompañante de toda acción humana resulta fundamental para comprender la unión indisoluble de los roles del interlocutor discursivo y del actor, que puede formularse del siguiente modo: el actor del mundo de la vida es ya un posible interlocutor. El concepto de discurso virtual acompañante permite superar una confusión compartida por las objeciones mencionadas. Esas objeciones confunden dos cosas bien distintas: por un lado, el hecho innegable de que los seres humanos pueden decidir arbitrariamente si entran, continúan o interrumpen un discurso y, por el otro, la problemática atribución a los agentes de la capacidad de decidir arbitrariamente si sus acciones pueden ser o no objeto de discurso. A diferencia de dichas objeciones, el sentido común parece no confundir las cosas de esta manera, porque la simple atribución de responsabilidad a un agente por su acción ya supone necesariamente la atribución de la capacidad de responder por su acción, esto es, la capacidad y la obligación de dar razones que la justifiquen.

Recibido: 03/2019; aceptado: 05/2019 